\title{
An integrated biochemical prediction model of all-cause mortality in patients undergoing lower extremity bypass surgery for advanced peripheral artery disease
}

\author{
Christopher D. Owens ${ }^{a}$, Ji Min Kim ${ }^{a}$, Nathanael D. Hevelone ${ }^{b, c}$, Warren J. Gasper ${ }^{a}$, Michael \\ Belkin $^{\mathrm{c}}$, Mark A. Creager ${ }^{\mathrm{C}}$, and Michael S. Conte ${ }^{\mathrm{a}}$ \\ aDivision of Vascular and Endovascular Surgery, University of California SanFrancisco \\ bThe Center for Surgery and Public Health \\ cThe Brigham and Women's Hospital
}

\section{Abstract}

Background-Patients with advanced peripheral artery disease (PAD) have a high prevalence of cardiovascular $(\mathrm{CV})$ risk factors and shortened life expectancy. However, CV risk factors poorly predict midterm $(<5$ years) mortality in this population. This study was designed to test the hypothesis that baseline biochemical parameters would add clinically meaningful predictive information in patients undergoing lower extremity bypass.

\begin{abstract}
Methods-This was a prospective cohort study of subjects with clinically advanced PAD undergoing lower extremity bypass surgery. The Cox proportional hazard was used to assess the main outcome of all-cause mortality. A clinical model was constructed with known cardiovascular risk factors and the incremental value of the addition of clinical chemistry, lipid, and a panel of 11 inflammatory parameters were investigated using c-statistic, the integrated discrimination improvement (IDI) index and Akaike information criterion (AIC).
\end{abstract}

Results-225 subjects were followed for a median 893 days; IQR 539-1315 days). In this study $50(22.22 \%)$ subjects died during the follow-up period. By life table analysis (expressed as percent surviving \pm standard error), survival at $1,2,3,4$, and 5 years respectively was $90.5 \pm 1.9 \%, 83.4 \pm$ $2.5 \%, 77.5 \pm 3.1 \%, 71.0 \pm 3.8 \%$, and $65.3 \pm 6.5 \%$. Compared with survivors, decedents were older, diabetic, had extant CAD, and were more likely to present with CLI as their indication for bypass surgery, $\mathrm{P}<.05$. After adjustment for the above, clinical chemistry and inflammatory parameters significant for all cause mortality were albumin, HR .43 (95\% CI .26-.71); P=.001, estimated glomerular filtration rate (eGFR), HR .98 (95\% CI .97-.99), P=.023, high sensitivity Creactive protein (hsCRP), HR 3.21 (95\% CI 1.21-8.55), $\mathrm{P}=.019$, and soluble vascular cell adhesion molecule (sVCAM), HR 1.74 (1.04-2.91), P=.034. Of all inflammatory molecules investigated, hsCRP proved most robust and representative of the integrated inflammatory response. Albumin, eGFR, and hsCRP improved the c-statistic and IDI beyond that of the clinical model and produced a final c-statistic of .82 .

(C) 2012 The Society for Vascular Surgery. Published by Mosby, Inc. All rights reserved.

Reprint requests: Christopher D. Owens MD, MSc, Division of Vascular and Endovascular Surgery, University of California San Francisco, 400 Parnassus Ave. San Francisco CA 94148.

Competition of interest: none

Publisher's Disclaimer: This is a PDF file of an unedited manuscript that has been accepted for publication. As a service to our customers we are providing this early version of the manuscript. The manuscript will undergo copyediting, typesetting, and review of the resulting proof before it is published in its final citable form. Please note that during the production process errors may be discovered which could affect the content, and all legal disclaimers that apply to the journal pertain. 
Conclusions-A risk prediction model including traditional risk factors and parameters of inflammation, renal function and nutrition had excellent discriminatory ability in predicting all cause mortality in patients with clinically advanced PAD undergoing bypass surgery.

\section{Introduction}

Patients undergoing arterial reconstructive procedures for advanced peripheral artery disease (PAD) tend to be elderly and have a high prevalence of cardiovascular risk factors including diabetes mellitus, hypertension, dyslipidemia, and tobacco abuse. Collectively, these risk factors are known to predict long-term mortality ( $\geq 10$ years) but are not sensitive at identifying patients at risk for death in the near- or mid-term, ( $\leq 5$ years).[1] Recently developed risk prediction models and scoring systems of one or two-year mortality in patients following lower extremity bypass surgery have revealed that patient-specific risk factors such as inadequate saphenous vein, the presence of critical limb ischemia, advanced age, or the Bollinger below knee angiogram score are the most potent independent predictors. [2-4] While these unique risk factors are unlikely to be responsible for death per se, they are most likely surrogates of the overall frailty of the patient.

It is known that patients with PAD have increased levels of inflammatory cytokines, [5] acute phase reactants, and soluble adhesion molecules, even after adjusting for age, gender, and traditional risk factors. [6] Elderly individuals with the highest levels of inflammatory biomarkers have increased short-term mortality ( $<5$ years). $[7,8]$ In the PAD population, inflammation, as assessed by high sensitivity C-reactive protein not only correlates directly with severity of PAD (higher in CLI patients) but is also associated with and death and cardiovascular events.[9-11]

The hypothesis of this study was that increased baseline inflammation would add clinically meaningful value to predicting all cause mortality in patients undergoing lower extremity bypass surgery. We a priori chose to examine a panel of 11 novel and diverse inflammatory biomarkers based on epidemiologic evidence implicating them with adverse cardiovascular outcomes and death. We sought to determine which biomarkers among these, if any, can add predictive value beyond that of pre- existing established risk factors for patients undergoing lower extremity bypass surgery.

\section{Methods}

\section{Study Design and population}

This was a National Institutes of Health funded prospective cohort study examining the relationship between inflammatory biomarkers and death following lower extremity bypass surgery. Three participating institutions underwent independent review of the study and received approval from the respective Institutional Review Boards. Each patient provided written informed consent. Enrollment began in February 2004 and ended in May 2008.

Details of patient selection/inclusion and exclusion criteria have been published previously. $[10,12,13]$ Patients were invited to participate in this study if planned to undergo a lower extremity bypass operation for disabling claudication or critical limb ischemia due to atherosclerotic obstructive disease. As the hypothesis to be tested was that baseline inflammatory and biochemical profile was predictive of mortality, we excluded anyone with active infection or concurrent illness that would cause a spurious increase in the plasma concentration of inflammatory biomarkers beyond the baseline state. Therefore our exclusion criteria included any evidence of active infection, pneumonia, malignancy, autoimmune disorders, or other precedent or concurrent significant illness 30 days prior to the index bypass procedure. While patients with small ulcers or small areas of dry gangrene 
were accepted into the study, deep space infections of the foot, those with large areas of ulceration, osteomyelitis, or ulceration or gangrene requiring operative debridement were excluded from participation. Patients on immunosuppressive medications or oral steroids were also excluded. As another aim of the present protocol involved the analysis of inflammation on vein graft-specific outcomes, only patients undergoing a bypass with autogenous vein were consented. In total, 225 patients were enrolled.

\section{Blood processing and assay measurements of biomarkers}

Plasma was collected with the patient in the fasting state on the morning of their bypass procedure. Blood was collected into EDTA and citrate vacutainer tubes and immediately iced. Tubes were spun at 3000 revolutions per minute for 20 minutes at $4^{\circ} \mathrm{C}$ in a refrigerated centrifuge. All samples were stored in a $-80^{\circ} \mathrm{C}$ freezer until the time of analysis. All analyses were conducted in batch at a core lab to avoid variation.

The Modification of Diet in Renal Disease Study equation was used to estimate glomerular filtration rate (eGFR) from serum creatinine (SCr): eGFR $\left(\mathrm{ml} \cdot \mathrm{min}^{-1} \cdot 1.73 \mathrm{~m}^{-2}\right)=175$. $(\mathrm{SCr})^{-1.154} \cdot(\text { age })^{-0.203} \cdot(0.742$ if female $) \cdot(1.210$ if black).[14] Body mass index (BMI) was expressed as $\mathrm{kg} / \mathrm{m}^{2}$.

\section{Clinical and endpoint definitions}

The endpoint investigated in this study was all cause mortality (death). Race, ethnicity, and gender were assessed by self-report. Subjects were considered to have hypertension if they were taking prescription medications for hypertension, if they had a systolic blood pressure $>140 \mathrm{mmHg}$ or a diastolic blood pressure $>90 \mathrm{mmHg}$. A diagnosis of hypercholesterolemia was present if they were taking prescription medications for cholesterol or if they self-report a prior diagnosis. Subjects were considered to have diabetes mellitus if the subject was taking prescription medications (oral or insulin) for diabetes, or if they self-reported a prior diagnosis. Subjects were considered to have coronary artery disease (CAD) if they had stable angina, prior myocardial infarction, prior coronary revascularization procedure, positive stress test, abnormal coronary angiogram, or ischemic cardiomyopathy. Active smoking was determined by self-report. Former smokers were defined as individuals who had smoked more than 100 cigarettes in their life but had not in the past 30 days and never smokers were defined as persons who had never smoked.

\section{Statistical analysis}

Baseline characteristics and biomarker values are presented as mean \pm SD or median and inter-quartile (IQR) range depending on the normality of their distribution. Proportions between groups were compared with $\mathrm{chi}^{2}$ test. Correlations were assessed with Pearson's correlation coefficients on log-normalized marker values. Univariate differences in plasma levels of markers between sub-groups of patients were analyzed with one-way nonparametric Wilcoxon rank sum (Mann-Whitney U) tests. Standard univariate analysis was performed between patient demographics, laboratory and lipid values, and inflammatory biomarkers and the primary outcome of death by log rank test. Inflammatory biomarkers were expressed as tertiles with the lowest tertile being the referent. In preliminary analysis, we explored multiple ways to express inflammatory markers and dividing them into tertile was most representative of discriminating mortality. The exception to this was hsCRP which we modeled as a dichotomized variable as we have done in the past. [10, 12]

Multivariable Cox proportional hazard modeling was performed on survival outcomes and hazard ratios and $95 \%$ confidence intervals are presented. A test of the proportional hazard assumption was performed for each covariate and globally using a formal significance test 
based on the unscaled and scaled Schoenfeld residuals. In the model construction, we followed the natural hierarchy of a decision making for the patient with PAD. First, a clinical model was constructed from all readily available clinical variables, including demographical data, medical history, and disease severity on presentation, i.e., CLI or claudication. This was noted as the clinical model and entry into the clinical model required a univariate $\mathrm{P}$ value $<.10$. We next determined the added benefit of clinical chemistry, lipid, and inflammatory parameters conditional on the clinical model. Due the number of inflammatory biomarkers examined, we broadly categorized this panel into three groups recognizing that there is considerable overlap among them. The three groups included factors involved in the coagulation and fibrinolytic system (PAI-1, tPA, and fibrinogen), soluble adhesion molecules and receptors (sVCAM-1, sICAM-1, sP-selectin, and sTNFR2), and the inflammatory cytokines and pentraxins (IL-6, MCP-1, hsCRP, and SAA). Soluble TNFR2 was used as a proxy for TNF-a, as it has been shown to be highly correlated with TNF-a, more stable in plasma, and more relevant for cardiovascular related outcomes. For these parameters, entry inclusion into the model required a univariate $\mathrm{P}$ value $<.10$ and a Harrell's c statistic $>.65$. This cutoff allowed retention of a diverse set of clinical and inflammatory parameters that we felt adequately represented the subject's biochemistry.

The ability to classify risk was assessed by the use of the Harrell's c statistic.[15] To determine the degree of risk reclassification the integrated discrimination improvement index (IDI) was employed. The IDI can be thought of as indicating how far individuals are moving on average along the continuum of predicted risk, i.e. how well a model separates subjects by the outcome of interest (death), by estimating the change in the difference in the mean predicted probabilities of the outcome between those with and without the outcome in question after introducing the candidate biomarker to the model.[16] Finally, we sought to obtain the most parsimonious model by minimizing the Akaike Information Criterion (AIC), which is a metric of the goodness-of-fit and accounts for the number of parameters required to obtain that degree of fitness. Statistical analyses were performed on Stata/SE 11.0 (StataCorp, College station, TX).

\section{Results \\ Population demographics}

The demographic summary of the study population is presented in Table 1 . The indication for bypass surgery was critical limb ischemia (CLI) in $133(59.1 \%)$ patients (Fontaine $2 b=92$, Fontaine $3=60$, and Fontaine $4=73$. The mean follow up from the index bypass procedure was 925 days (median 893; IQR 539-1315 days). No surviving individual was followed less than 365 days. Fifty (22.2\%) subjects died during the follow-up period. By life table analysis (expressed as percent surviving \pm standard error), survival at $1,2,3,4$, and 5 years respectively was $90.5 \pm 1.9 \%, 83.4 \pm 2.5 \%, 77.5 \pm 3.1 \%, 71.0 \pm 3.8 \%$, and $65.3 \pm$ $6.5 \%$. There were two deaths within 30 days and 28 deaths prior to one year. Compared with survivors, decedents were older (mean age 71.7 years [SD, 10.7] vs. 66.5 years [10.8]; $\mathrm{P}=$. 003), had a higher incidence of established CAD (66\% vs. 49\%; $\mathrm{P}=.030)$ and were more likely to present with CLI as their indication for bypass surgery ( $80 \%$ vs. 53. \%; $\mathrm{P}=.001$ ) (Table 1). The use of a statin at the time of bypass was not associated with survival however only $45(20 \%)$ patients were not on statins and there were only 7 deaths among these individuals during follow up.

The bypass graft characteristics and outcomes have been published elsewhere.[10, 12, 17] In brief, 202 bypass grafts were constructed from single segment or composite great saphenous vein and 23 were from arm vein. There were 111 femoral-popliteal, 78 femoral-tibial, and 36 femoral or popliteal-pedal bypass grafts. The 2-year primary and secondary patency rates were $61.4 \pm 3.6 \%$ and $83.1 \pm 2.7 \%$, respectively. 
Albumin, hemoglobin and eGFR were all significantly lower in the CLI subgroup, (Table 2). As expected, most biomarkers of inflammation were higher in subjects presenting with CLI compared to those presenting with claudication (Table 3). However, there was no difference in the WBC (Table 2). The finding of no difference in WBC between CLI and claudication subjects is expected given our stringent exclusion criteria for entry into this study. Both total cholesterol and LDL cholesterol as well as apolipoprotein A1 and B100 were lower in patients in subjects presenting with CLI (Table 2).

\section{Biomarkers and death}

Baseline albumin, hemoglobin, eGFR, and total cholesterol and LDL cholesterol were lower in decedents than in those patients surviving $(\mathrm{P}<.05, \log$ rank, Table 2$)$. In particular albumin $(3.37 \pm .60$ vs. $3.67 \pm .46 ; \mathrm{P}=.0009)$ and eGFR (59.5 \pm 35.9 vs. $80.8 \pm 33.2 ; \mathrm{P}=$. 0001) were significantly lower in patients who subsequently died compared with those who survived. By contrast, baseline levels of all inflammatory cytokines and pentraxins, fibrinogen, and all soluble receptors and cell adhesion molecules except sP-selectin were markedly elevated in patients who subsequently died (Table 3). All biomarkers were positively and significantly correlated with one another and negatively correlated with albumin ( $\mathrm{P}<.0002$ for all correlations). The Pearson correlation coefficient between albumin and total cholesterol was $.27, \mathrm{P}=.0006$, reflecting the nutritionally depleted state in subjects who subsequently died during follow up. However, there was no correlation between albumin and the patient's BMI $(\mathrm{P}=.07)$.

Table 4 shows the univariate association expressed as hazard ratio (HR) and 95\% CI as well as the c-statistic between patient demographics, laboratory and lipid values with all cause mortality. All biomarkers were modeled as tertiles with the exception of CRP. The biomarker CRP was modeled as a dichotomized variable with a cutoff of $5 \mathrm{mg} / \mathrm{L}$. We chose to do this because our previous work has shown that CRP offers the best ability to discriminate between patients undergoing lower extremity bypass surgery into those with and without adverse graft-related events.[10] Further, patients with CRP levels above $5 \mathrm{mg}$ / L exhibit impaired vein graft remodeling compared to patients with lower levels of CRP, implying that this value is clinically relevant in terms of vein graft-related outcomes.[12] In addition, the sensitivity and specificity of hsCRP $>5 \mathrm{mg} / \mathrm{L}$ for subsequent midterm mortality were $70.2 \%$ and $72.0 \%$, respectively. This represents the highest sum of sensitivity and specificity and therefore is an optimal cutoff for this analyte. By univariate analysis, hsCRP levels $>5 \mathrm{mg} / \mathrm{L}$ was associated with increased mortality (HR 4.54, 95\% CI 2.43$8.49, \mathrm{P}<0.0001)$, Figure 1.

In multivariable analysis, clinical risk factors meeting inclusion criteria included age, diabetes mellitus, the presence of established CAD, and CLI as an indication for bypass. Clinical and inflammatory biomarker values meeting inclusion criteria included albumin, eGFR, hsCRP, sVCAM-1, sTNFR2 and fibrinogen (Table 5). Representative biomarkers from each of the three broad categories were present in the final model. Although IL-6 formally met the inclusion criteria into the final model, it was omitted given its known role of induction of CRP production.[18] Lower tertiles of total cholesterol, LDL, HDL, and apolipoprotein A1 and B100 were associated with decreased survival on univariate analysis; however, no lipid parameter met inclusion criteria for the final model. The hazard ratios and $95 \% \mathrm{CI}$ of all variables entered into the final model are presented in table 5. Higher eGFR [HR=.98 (95\%CI .97-.99); $\mathrm{P}=.023]$ and albumin [HR=.43 (95\%CI .26-.71); $\mathrm{P}=.001]$ were protective for overall death while higher hsCRP $>5 \mathrm{mg} / \mathrm{L}$ [HR=3.21 (95\%CI 1.21-8.55); $\mathrm{P}=.019]$ and sVCAM-1 [HR=1.74 (95\%CI 1.04-2.91); $\mathrm{P}=.034$ were predictive of mortality. The hazard of reduced serum albumin was linear through the full range of albumin, 2.2-4.9 $\mathrm{mg} / \mathrm{dl}$. Additionally, albumin predicted death equally well for patients with high levels of inflammatory biomarkers (hsCRP>5 mg/l), $\mathrm{HR}=.48(95 \% \mathrm{CI} .28-.83)$ as it does for 
patients with hsCRP< $5 \mathrm{mg} / \mathrm{L}, \mathrm{HR}=.49$ (95\% CI .29-.84), indicating an effect size that is independent of the inflammatory state of the subject. While the HR for each unit increase of eGFR was marginal at .98, it is well known that the hazard for reduced renal function begins well before the onset of hemodialysis.[19] In the present analysis, the hazard ratio for each standard deviation, $35 \mathrm{ml} \cdot \min ^{-1} \cdot 1.73 \mathrm{~m}^{-2}$, increase in eGFR was .54 .

The clinical model produced a c-statistic of .71 (Table 6). When eGFR, albumin, and hsCRP were added to the model the c-statistic was increased to .82 . Among all biochemical parameters investigated, albumin contributed the largest increase in the c-statistic beyond the clinical model, .09. The addition of hsCRP to the clinical model plus eGFR and albumin improved the c-statistic by .02. Importantly, the IDI, an assessment of incremental improvement in risk discrimination after addition of a new predictor, for the addition of eGFR, albumin, and hsCRP respectively to the clinical model were all highly significant (table 6). The addition of any other clinical or inflammatory marker (sVCAM, sTNFR2) failed to significantly improve the discriminatory ability of the final model consisting of age, diabetes, CAD, CLI, albumin, egfr, and hsCRP.

\section{Discussion}

Management decisions for patients with severe PAD are based on multiple levels of information processed in a natural hierarchical order. For example, demographic characteristics, prevalent and previous medical history, and clinical presentation are considered first followed by the consideration of routine laboratory and angiographic tests. The addition of a biomarker must be predicated on its ability to clinical care above that provided by routine history, exam, and laboratory tests. For any risk model to have practical value, it must be intuitive and easy to incorporate into clinical practice. Herein, the final model constructed contains a diverse set of variables including traditional demographics such as age and diabetes, severity of PAD, a measure of renal function, an assessment of protein-energy malnutrition, and a marker of inflammation. The 7 parameters represented in the final model are naturally intuitive and readily obtainable in a physician's office; hence its practical value. In addition, the final model has a c statistic of .82 which is within the range considered to have excellent discriminatory ability.[20] A c-statistic of .82 indicates that the model can adequately discriminate between a randomly selected patient who died during followup from a randomly selected patient who survived, $82 \%$ of the time. Finally the model minimizes the AIC which is a metric of goodness-of-fit and complexity as it imposes a penalty for the number of parameters needed to achieve the particular degree of fitness. It is a quantification of model preference seeking the fewest explanatory variables required to provide adequate fit to the data.

One of the immediate observations of the multivariable model is that traditional risk factors, including cholesterol fractions and smoking, were not significantly associated with the primary endpoint of midterm death in this cohort. Rather, impaired renal function, malnutrition, and inflammation were the most powerful predictors. This is consistent with previous investigations which have shown markers of inflammation are better predictors of short and midterm mortality while traditional cardiovascular risk factors are better in the long term ( $\geq 10$ years).[7, 11, 21, 22] Models incorporating more proximate causes of midterm death are desirable not only to inform management decisions but allow for a potentially mitigating intervention. Identification of a high-risk patient may encourage the practitioner to choose a less invasive alternative to bypass such as endovascular revascularization. Understanding midterm survival seems especially prudent in light of the recently completed Bypass versus Angioplasty for Severe Ischemia of the Leg (BASIL) trial demonstrating an overall survival advantage in patients randomized to bypass surgery in patients who survived at least 2 years after the bypass.[2] 
We felt it was unlikely that one marker could adequately represent the total inflammatory burden and that the simultaneous measurement of a panel of biomarkers would more adequately represent the integrated inflammatory risk. Thus we measured 11 novel markers from 3 broad categories, thrombotic/fibrinolytic, soluble cell surface receptors and adhesion molecules, and inflammatory cytokines and acute phase reactants. However, the addition of hsCRP to the full clinical model plus eGFR and albumin essentially quenched the model from further contribution of any other inflammatory biomarker. Whereas, sVCAM retained statistical significance in the multivariable model, no additional clinically meaningful information, as determined by the C-statistic or the IDI index, was gained from its inclusion. In this regard, hsCRP has proved the most robust marker for inflammation in this cohort of patients undergoing lower extremity bypass surgery.

The inclusion of the full biomarker panel however, is heuristic because enrolled subjects, particularly the ones who died during follow up, had a marked elevation in proinflammatory cytokines such as sTNFR2 and IL-6. These cytokines elicit an acute phase response that has profound nutritional implications.[23] It is energy-intensive with high rates of hepatic protein synthesis and requires large quantities of essential amino acids. Through a variety of described pathways, [24] long term exposure to elevated levels of cytokines enhances protein catabolism and induces anorexia, and lean muscle mass is expended to support synthesis of acute phase proteins. The culmination of these processes creates a state of vascular cachexia. Indeed, the values of IL-6 and sTNFR2 in subjects who died during follow up are comparable to patients with advanced stage congestive heart failure and patients with known cardiac cachexia.[25, 26] Similar to other forms of non-malignant cachexia, this is a chronic hypercytokinemic cachexia with relative preservation of body weight and BMI in most patients.[27] For example, only 32 patients in this study had a BMI $<22 \mathrm{~kg} \cdot \mathrm{m}^{-2}$, and no correlation existed between BMI and albumin. Along with albumin, there is also reduced hepatic production of lipoproteins associated with the inflammatory/ malnourished state.[28] Cholesterol fractions and both apolipoprotein A1 and B100 were decreased in patients who died in this study. However, the seemingly paradoxical finding that lower total cholesterol, HDL, LDL, apoA1, and apoB100 had significant univariate association with death was mitigated by inclusion of inflammatory, nutritional, and renal parameters. Therefore, in this study, like others, the so-called J-shaped association between lipid parameters and mortality is probably a result of confounding.[29]

The combined effect of inflammation, renal failure and malnutrition has received little attention in patients undergoing lower extremity bypass despite the considerable overlap among them. Synergism of each to the hazard of death can be demonstrated by Venn diagrams, with relative malnourishment (albumin $<3.5 \mathrm{mg} / \mathrm{dl} ; \mathrm{N}=74$ ), renal impairment (egfr $<60 \mathrm{ml} / \mathrm{min} ; \mathrm{N}=63$ ) and high levels of inflammation (hsCRP> $5 \mathrm{mg} / \mathrm{l} ; \mathrm{N}=80$ ) represented (figure 2). This is particularly alarming as the hazard ratio for death for the $21(9 \%)$ subjects located at the convergence of all three circles was 9.08 (95\% CI 4.8517.01). Therefore the clinical consequence of even modest impairment of more than one of these 3 domains is substantial.

\section{Limitations}

Limitations in this study include the fact that the cohort consisted predominantly of elderly white men. Further studies are needed to determine whether this model improves risk stratification in women, in younger persons, and in other ethnic groups. Some biomarker values in this study were extremely elevated. Because of the strict exclusion criteria applied and to the best of our knowledge, the elevated marker values likely reflect the true baseline state of the patient rather than spurious elevation due to concurrent illness. Albumin was used as a nutritional index in this examination which has a half-life of approximately 20 days. It is possible that another metric of nutrition would have been more appropriate such 
as transthyretin, prealbumin, (half-life $=2$ days) which is more sensitive to changes in protein-energy status than albumin.[30] However, while transthyretin accurately reflects recent nitrogen intake, albumin may better represent the overall baseline nutritional state of the patient.[31] Finally, this study was modest in size and results must be considered with caution until external validation studies can be undertaken.

\section{Implications of the study}

Several methods including c-statistic, IDI, and AIC were used to provide diagnostic information about model iterations. Together these measures not only quantify calibration and discrimination but also ensure that the most parsimonious model is used to best fit the data. A model incorporating biochemical parameters has theoretical advantages to guide potential treatment strategies to improve survival among high risk subjects. Antiinflammatory or anti-cytokine therapy may mitigate some of the effects of the acute phase response seen in this population. $[32,33]$ Nutritional therapy with $\omega-3$ fatty acids, appetite stimulants, or metabolic regulators have shown promise in similar cohorts and may improve the recovery period following surgery and improve functional capacity.[34, 35] Progressive resistive training has been shown to improve lean mass as well as biochemical parameters in patients with high levels of inflammation. It is currently unknown whether any of these therapies could improve outcomes in the clinically-advanced PAD population.

We conclude that the risk model presented herein containing parameters of inflammation, nutrition, and renal function, has excellent discriminatory ability for midterm all-cause mortality in patients selected to undergo lower extremity arterial bypass surgery. Among all markers of inflammation, hsCRP has proven the most robust and is most representative of the integrated inflammatory response. A significant minority of patients undergoing lower extremity bypass surgery have a hypercytokinemic, hypoalbuminemic vascular cachexia, placing them at particular increased risk for death. Whether there are any therapeutic mitigating strategies to decrease the inflammatory response, or improve the biochemical parameters in these high risk patients remains to be tested.

\section{References}

1. Stamler J, et al. Low risk-factor profile and long-term cardiovascular and noncardiovascular mortality and life expectancy: findings for 5 large cohorts of young adult and middle-aged men and women. Jama. 1999; 282(21):2012-8. [PubMed: 10591383]

2. Bradbury AW, et al. Bypass versus Angioplasty in Severe Ischaemia of the Leg (BASIL) trial: A survival prediction model to facilitate clinical decision making. J Vasc Surg. 2010; 51(5 Suppl): 52S-68S. [PubMed: 20435262]

3. Goodney PP, et al. Factors associated with death 1 year after lower extremity bypass in Northern New England. J Vasc Surg. 2010; 51(1):71-8. [PubMed: 19939615]

4. Schanzer A, et al. Risk stratification in critical limb ischemia: derivation and validation of a model to predict amputation-free survival using multicenter surgical outcomes data. J Vasc Surg. 2008; 48(6):1464-71. [PubMed: 19118735]

5. DePalma RG, et al. Cytokine signatures in atherosclerotic claudicants. J Surg Res. 2003; 111(2): 215-21. [PubMed: 12850465]

6. McDermott MM, et al. Patterns of inflammation associated with peripheral arterial disease: the InCHIANTI study. Am Heart J. 2005; 150(2):276-81. [PubMed: 16086930]

7. Vidula $\mathrm{H}$, et al. Biomarkers of inflammation and thrombosis as predictors of near-term mortality in patients with peripheral arterial disease: a cohort study. Ann Intern Med. 2008; 148(2):85-93. [PubMed: 18195333]

8. Jenny NS, et al. Inflammation biomarkers and near-term death in older men. Am J Epidemiol. 2007; 165(6):684-95. [PubMed: 17215383] 
9. Vainas T, et al. C-reactive protein in peripheral arterial disease: relation to severity of the disease and to future cardiovascular events. J Vasc Surg. 2005; 42(2):243-51. [PubMed: 16102622]

10. Owens CD, et al. Elevated C-reactive protein levels are associated with postoperative events in patients undergoing lower extremity vein bypass surgery. J Vasc Surg. 2007; 45(1):2-9. discussion 9. [PubMed: 17123769]

11. Barani $\mathrm{J}$, et al. Inflammatory mediators are associated with 1-year mortality in critical limb ischemia. J Vasc Surg. 2005; 42(1):75-80. [PubMed: 16012455]

12. Owens $\mathrm{CD}$, et al. Early remodeling of lower extremity vein grafts: inflammation influences biomechanical adaptation. J Vasc Surg. 2008; 47(6):1235-42. [PubMed: 18440187]

13. Owens CD, et al. Novel adipokines, high molecular weight adiponectin and resistin, are associated with outcomes following lower extremity revascularization with autogenous vein. J Vasc Surg. 51(5):1152-9. [PubMed: 20223619]

14. Levey AS, et al. A more accurate method to estimate glomerular filtration rate from serum creatinine: a new prediction equation. Modification of Diet in Renal Disease Study Group. Ann Intern Med. 1999; 130(6):461-70. [PubMed: 10075613]

15. Harrell FE Jr, Lee KL, Mark DB. Multivariable prognostic models: issues in developing models, evaluating assumptions and adequacy, and measuring and reducing errors. Stat Med. 1996; 15(4): 361-87. [PubMed: 8668867]

16. Pencina MJ, et al. Evaluating the added predictive ability of a new marker: from area under the ROC curve to reclassification and beyond. Stat Med. 2008; 27(2):157-72. discussion 207-12. [PubMed: 17569110]

17. Owens CD, et al. Novel adipokines, high molecular weight adiponectin and resistin, are associated with outcomes following lower extremity revascularization with autogenous vein. J Vasc Surg.

18. Moshage HJ, et al. The effect of interleukin-1, interleukin-6 and its interrelationship on the synthesis of serum amyloid A and C-reactive protein in primary cultures of adult human hepatocytes. Biochem Biophys Res Commun. 1988; 155(1):112-7. [PubMed: 3261980]

19. Owens CD, et al. Refinement of survival prediction in patients undergoing lower extremity bypass surgery: stratification by chronic kidney disease classification. J Vasc Surg. 2007; 45(5):944-52. [PubMed: 17391900]

20. Lloyd-Jones DM. Cardiovascular risk prediction: basic concepts, current status, and future directions. Circulation. 2010; 121(15):1768-77. [PubMed: 20404268]

21. Criqui $\mathrm{MH}$, et al. Biomarkers in peripheral arterial disease patients and near- and longer-term mortality. J Vasc Surg. 2010; 52(1):85-90. [PubMed: 20471776]

22. Doweik L, et al. Fibrinogen predicts mortality in high risk patients with peripheral artery disease. Eur J Vasc Endovasc Surg. 2003; 26(4):381-6. [PubMed: 14511999]

23. Morley JE, Thomas DR, Wilson MM. Cachexia: pathophysiology and clinical relevance. Am J Clin Nutr. 2006; 83(4):735-43. [PubMed: 16600922]

24. Saini A, Al-Shanti N, Stewart CE. Waste management - cytokines, growth factors and cachexia. Cytokine Growth Factor Rev. 2006; 17(6):475-86. [PubMed: 17118696]

25. Anker SD, et al. Cytokines and neurohormones relating to body composition alterations in the wasting syndrome of chronic heart failure. Eur Heart J. 1999; 20(9):683-93. [PubMed: 10208789]

26. Deswal A, et al. Cytokines and cytokine receptors in advanced heart failure: an analysis of the cytokine database from the Vesnarinone trial (VEST). Circulation. 2001; 103(16):2055-9. [PubMed: 11319194]

27. Walsmith $\mathbf{J}$, et al. Tumor necrosis factor-alpha production is associated with less body cell mass in women with rheumatoid arthritis. J Rheumatol. 2004; 31(1):23-9. [PubMed: 14705214]

28. Myasoedova E, et al. Lipid paradox in rheumatoid arthritis: the impact of serum lipid measures and systemic inflammation on the risk of cardiovascular disease. Ann Rheum Dis. 2011; 70(3):482-7. [PubMed: 21216812]

29. Hu P, et al. Does inflammation or undernutrition explain the low cholesterol-mortality association in high-functioning older persons? MacArthur studies of successful aging. J Am Geriatr Soc. 2003; 51(1):80-4. [PubMed: 12534850]

30. Shenkin A. Serum prealbumin: Is it a marker of nutritional status or of risk of malnutrition? Clin Chem. 2006; 52(12):2177-9. [PubMed: 17138848] 
31. Ingenbleek Y V, Young R. Significance of transthyretin in protein metabolism. Clin Chem Lab Med. 2002; 40(12):1281-91. [PubMed: 12553432]

32. Ridker PM. Testing the inflammatory hypothesis of atherothrombosis: scientific rationale for the cardiovascular inflammation reduction trial (CIRT). J Thromb Haemost. 2009; 7(Suppl 1):332-9. [PubMed: 19630828]

33. Marcora SM, et al. Randomized phase 2 trial of anti-tumor necrosis factor therapy for cachexia in patients with early rheumatoid arthritis. Am J Clin Nutr. 2006; 84(6):1463-72. [PubMed: 17158431]

34. Akner G, Cederholm T. Treatment of protein-energy malnutrition in chronic nonmalignant disorders. Am J Clin Nutr. 2001; 74(1):6-24. [PubMed: 11451713]

35. Kotler DP. Cachexia. Ann Intern Med. 2000; 133(8):622-34. [PubMed: 11033592]

$J$ Vasc Surg. Author manuscript; available in PMC 2013 September 01. 


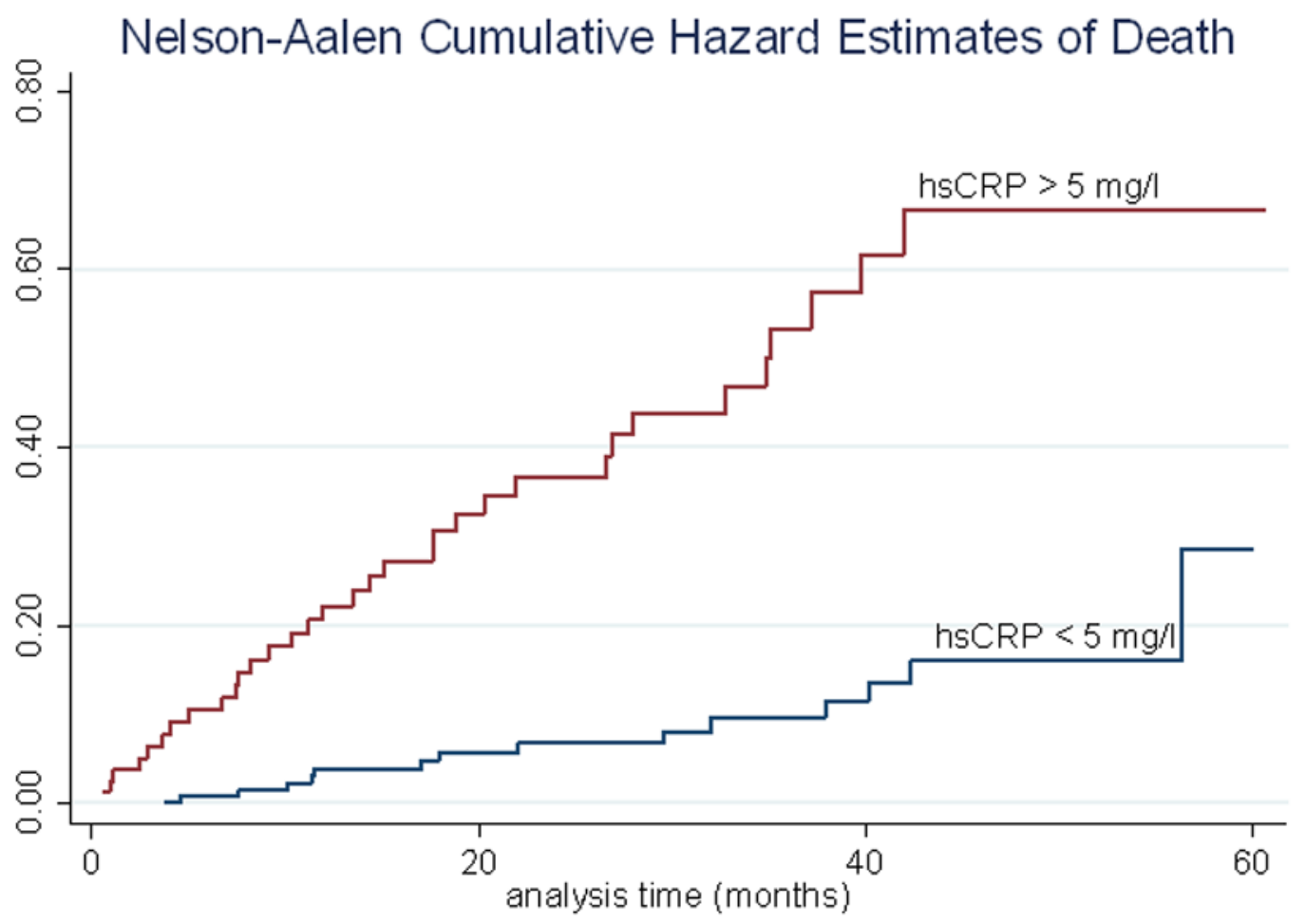

Figure 1.

Nelson Aalen cumulative hazard estimates for subjects dichotomized by pre-operative baseline hsCRP values above or below $5.0 \mathrm{mg} / \mathrm{l}$. 


\section{Malnutrition (albumin $<3.5 \mathrm{mg} / \mathrm{dl}$ ) Renal impairment (eGFR $<60 \mathrm{ml} / \mathrm{min}$ )}

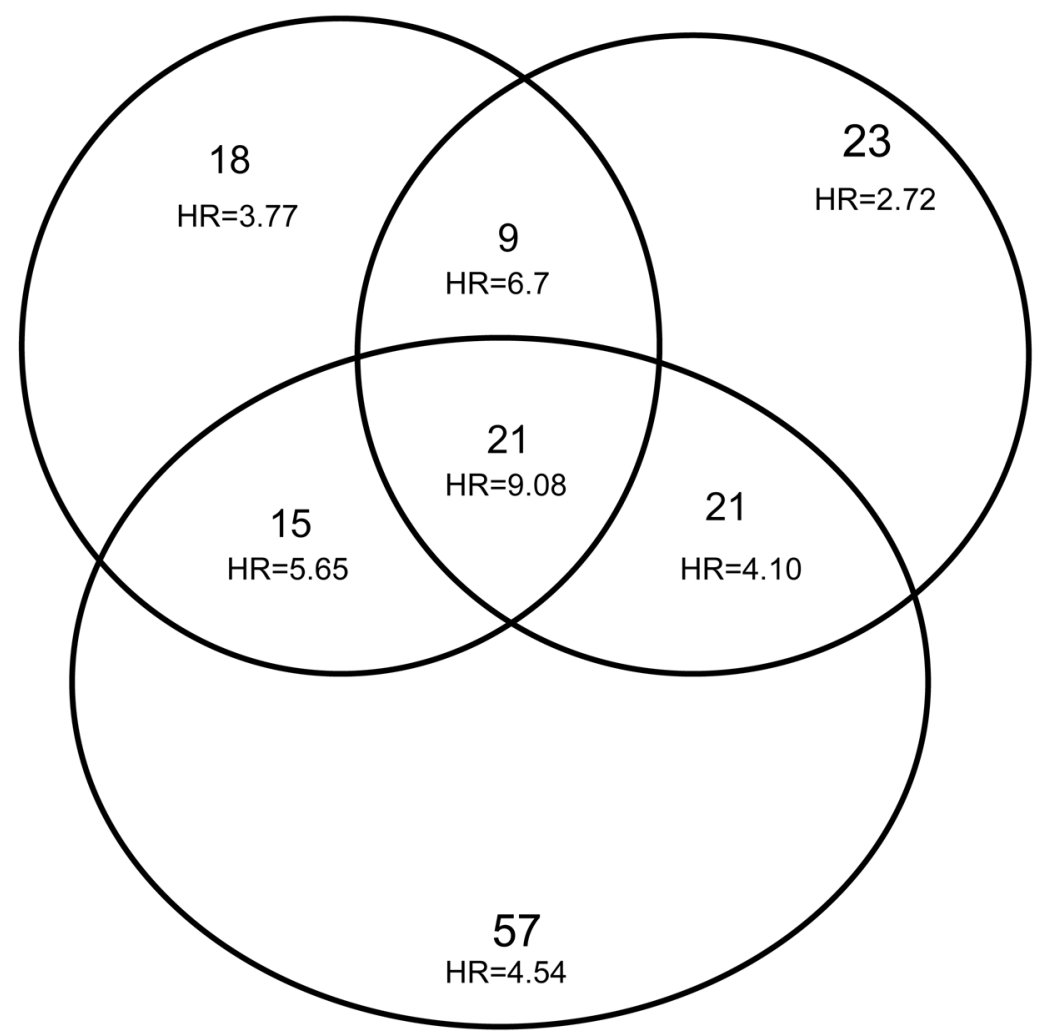

Inflammation (hsCRP> 5mg/L)

Figure 2.

Venn diagram demonstrating the overlap among subsets of subjects with malnutrition, renal impairment, and inflammation and the synergism of different mechanisms for the hazard of death. Out of 225 subjects in this study, $74(33 \%)$ had an eGFR $<60 \mathrm{ml} \cdot \mathrm{min}^{-1} \cdot 1.73 \mathrm{~m}^{-2}$, $63(28 \%)$ had an albumin $<3.5 \mathrm{mg} / \mathrm{dl}$, and $80(36 \%)$ had a hsCRP > $5.0 \mathrm{mg} / \mathrm{l}$. The $21(9 \%)$ subjects located within the intersection of all 3 circles have a hazard ratio for death of 9.08 . 
Table 1

Demographic characteristics of the study population

\begin{tabular}{|l|l|l|l|l|}
\hline Demographic variable & Overall $(\mathbf{N}=\mathbf{2 2 5})$ & Alive $(\mathbf{N = 1 7 5})$ & Dead $(\mathbf{N = 5 0 )}$ & P-value \\
\hline Age $($ Mean \pm SD) & $67.62 \pm 10.93$ & $66.47 \pm 10.75$ & $71.66 \pm 10.69$ & .0029 \\
\hline Gender $(\mathrm{male}, \%)$ & $161(71.56 \%)$ & $127(72.57)$ & $34(68.0)$ & .527 \\
\hline BMI $\left(\mathrm{kg} \cdot \mathrm{m}^{-2}\right)$ & $28.36 \pm 6.51$ & $28.27 \pm 6.18$ & $28.70 \pm 7.64$ & .6804 \\
\hline Hypertension $(\%)$ & $190(84.4 \%)$ & $146(83.43)$ & $44(88.00)$ & .432 \\
\hline CAD $(\%)$ & $118(52.4 \%)$ & $85(48.57)$ & $33(66.00)$ & .030 \\
\hline Hyperlipidemia $(\%)$ & $162(72.0 \%)$ & $125(71.43)$ & $37(74.00)$ & .721 \\
\hline White $(\%)$ & $196(87.1 \%)$ & $149(85.14)$ & $47(94.00)$ & .099 \\
\hline CLI $(\%)$ & $133(59.1 \%)$ & $93(53.14)$ & $40(80.00)$ & .001 \\
\hline Diabetes $(\%)$ & $117(52.0 \%)$ & $85(48.57)$ & $32(64.00)$ & .054 \\
\hline $\begin{array}{l}\text { Tobacco }(\%) \\
\text { Former } \\
\text { Current }\end{array}$ & $98(43.6 \%)$ & $77(44.00)$ & $21(42.00)$ & .801 \\
\hline Statin use $(\%)$ & $63(36.9 \%)$ & $65(37.14)$ & $18(36.00)$ & .883 \\
\hline Anti-platelet use (\%) & $182(81 \%)$ & $142(81.14)$ & $40(80.00)$ & .545 \\
\hline ACE/ARB use (\%) & $126(56 \%)$ & $104(59.43)$ & $22(48.00)$ & .175 \\
\hline B-blocker use & $183(81.3)$ & $141(80.57)$ & $42(84.00)$ & .993 \\
\hline
\end{tabular}




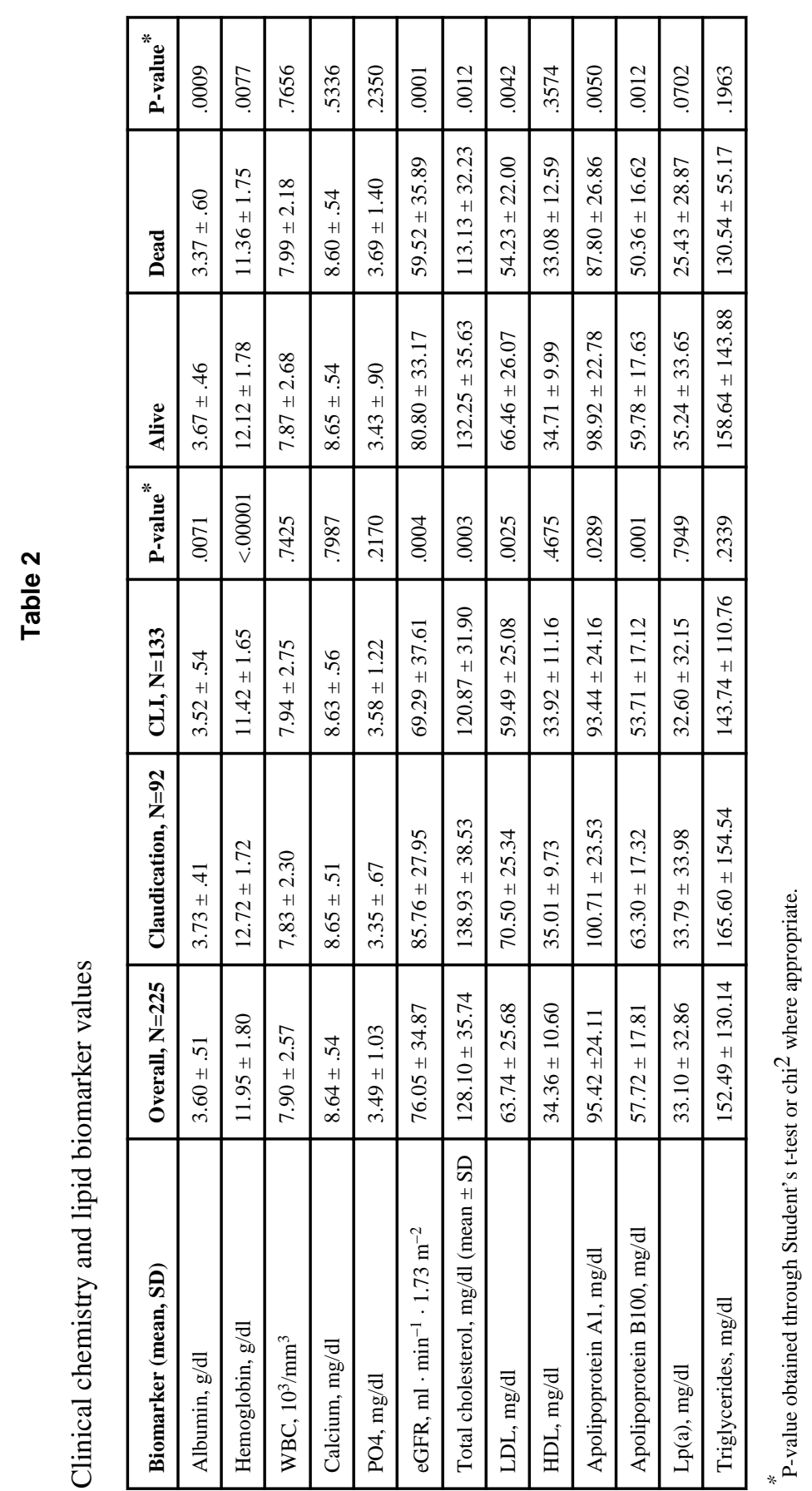




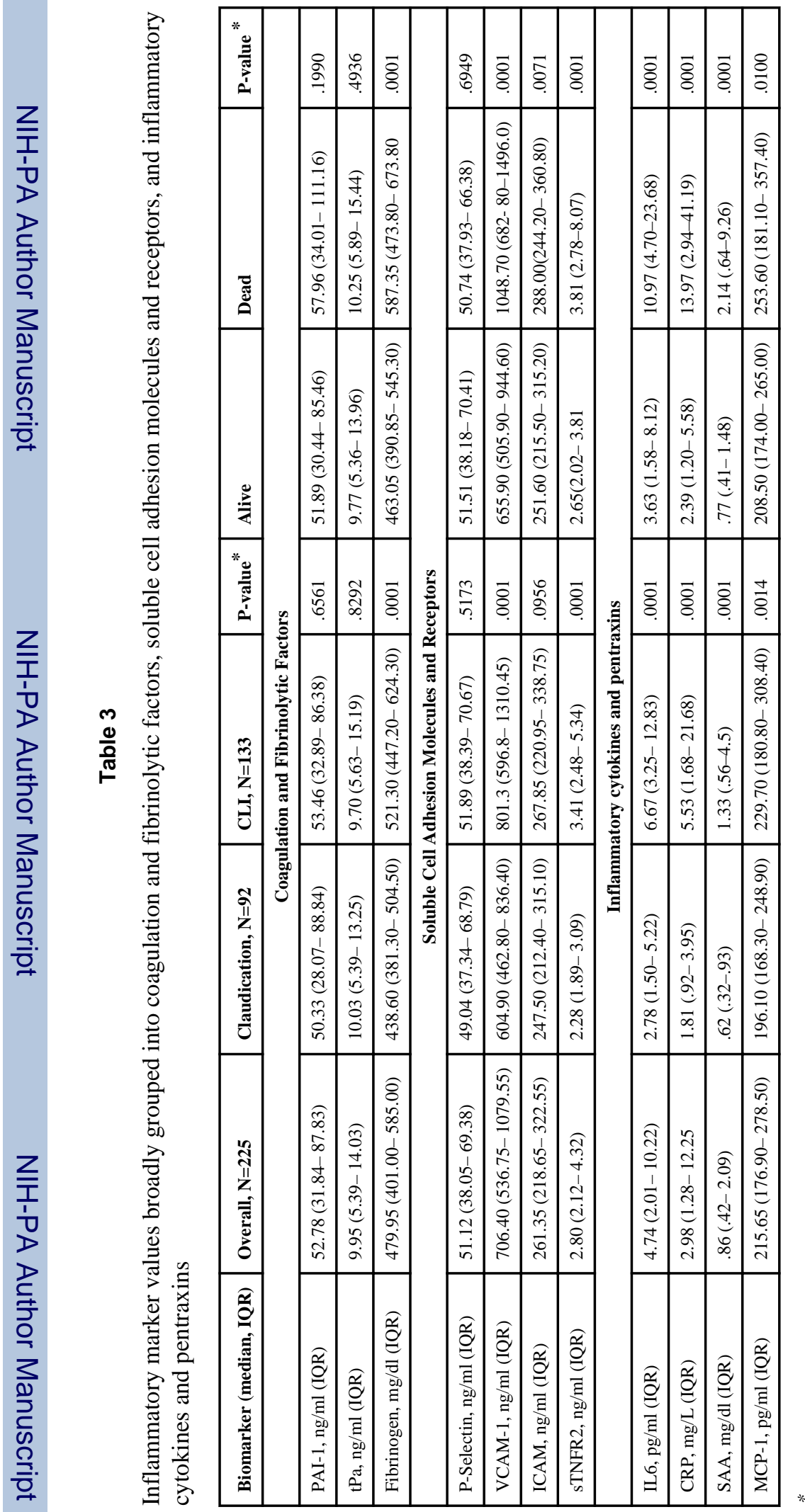

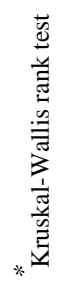


Table 4

Univariate association of demographic, clinical, and lipid parameters with all cause mortality.

\begin{tabular}{|c|c|c|c|}
\hline Predictor variables & HR $(95 \%$ CI) for Death & P-value & C-statistic \\
\hline \multicolumn{4}{|c|}{ Baseline demographics and disease presentation } \\
\hline Age & $1.04(1.01-1.07)$ & .003 & .6288 \\
\hline Gender (male) & $.85(.46-1.54)$ & .598 & .5146 \\
\hline Race (white) & $.62(.29-1.32)$ & .224 & .5335 \\
\hline Diabetes Mellitus & $1.69(.95-3.01)$ & .074 & .5618 \\
\hline CAD & $1.96(1.09-3.53)$ & .023 & .6002 \\
\hline Hypertension & $1.44(.61-3.38)$ & .401 & .5152 \\
\hline Hyperlipidemia & $1.08(.57-2.04)$ & .791 & .5114 \\
\hline Tobacco (current) & $.77(.43-1.37)$ & .379 & .5352 \\
\hline CLI & $3.35(1.67-6.71)$ & .001 & .6358 \\
\hline \multicolumn{4}{|c|}{ Clinical laboratory values } \\
\hline *Albumin & $.37(.24-.58)$ & $<.0001$ & .7049 \\
\hline *Hemoglobin & $.53(.37-.78)$ & .001 & .6213 \\
\hline *WBC & $1.13(.79-1.62)$ & .479 & .5397 \\
\hline Calcium & $.73(.43-1.23)$ & .242 & .5655 \\
\hline PO4 & $1.04(.75-1.45)$ & .789 & .4297 \\
\hline eGFR & $.98(.97-.99)$ & $<.0001$ & .6503 \\
\hline \multicolumn{4}{|c|}{ Lipid values } \\
\hline *Total cholesterol & $.62(.43-.91)$ & .014 & .6218 \\
\hline$* \mathrm{LDL}$ & $.59(.40-.86)$ & .007 & .6415 \\
\hline *HDL & $.63(.43-.92)$ & .018 & .6178 \\
\hline *Apolipoprotein A1 & $.66(.45-.96)$ & .034 & .5984 \\
\hline *Apolipoprotein B100 & $.62(.43-90)$ & .013 & .6158 \\
\hline$* \operatorname{Lp}(a)$ & $.84(.59-1.19)$ & .351 & .5386 \\
\hline *Triglycerides & $1.06(.75-1.50)$ & .727 & .4982 \\
\hline \multicolumn{4}{|c|}{ Fibrinolytic Factors } \\
\hline PAI-1 & $1.19(.83-1.70)$ & .332 & .5395 \\
\hline $\mathrm{t}-\mathrm{Pa}$ & $1.04(.73-1.49)$ & .792 & .5305 \\
\hline Fibrinogen & $2.23(1.50-3.33)$ & $<.0001$ & .6810 \\
\hline \multicolumn{4}{|c|}{ Soluble Cell Adhesion Molecules and receptors } \\
\hline sP-Selectin & $.93(.65-1.32)$ & .705 & .5061 \\
\hline SVCAM-1 & $2.15(1.45-3.20)$ & $<.0001$ & .6824 \\
\hline sICAM-1 & $1.67(1.16-2.42)$ & .006 & .6452 \\
\hline sTNFR2 & $1.93(1.32-2.83)$ & .001 & .6572 \\
\hline \multicolumn{4}{|c|}{ Inflammatory cytokines and pentraxins } \\
\hline IL-6 & $2.37(1.58-3.56)$ & $<.0001$ & .6943 \\
\hline hsCRP > $5 \mathrm{mg} / \mathrm{L}$ & $4.54(2.43-8.49)$ & $<.0001$ & .7001 \\
\hline
\end{tabular}




\begin{tabular}{|l|l|l|l|}
\hline Predictor variables & HR $(\mathbf{9 5 \%}$ CI) for Death & P-value & C-statistic \\
\hline MCP-1 & $1.43(1.00-2.06)$ & .049 & .5759 \\
\hline SAA & $1.90(1.29-2.81)$ & .001 & .6453 \\
\hline
\end{tabular}


Table 5

\begin{tabular}{|l|l|l|l|}
\hline & Parameter estimate (SE) & Hazard ration (95\% CI) & p-value \\
\hline Age & $-.003 \pm .017$ & $.99(.96-1.03)$ & .859 \\
\hline Diabetes & $.125 \pm .410$ & $1.10(.50-2.41)$ & .807 \\
\hline CAD & $.490 \pm .412$ & $1.59(.72-3.52)$ & .249 \\
\hline CLI & $.614 \pm .475$ & $1.86(.73-4.73)$ & .192 \\
\hline eGFR & $-.013 \pm .006$ & $.98(.97-.99)$ & .023 \\
\hline hsCRP> 5mg/l & $1.15 \pm .499$ & $3.21(1.21-8.55)$ & .019 \\
\hline Albumin (tertiles) & $-.835 \pm .254$ & $.43(.26-.71)$ & .001 \\
\hline sVCAM-1 (tertiles) & $.595 \pm .300$ & $1.74(1.04-2.91)$ & .034 \\
\hline sTNFR2 (tertiles) & $-.101 \pm .341$ & $.90(.46-1.76)$ & .767 \\
\hline Fibrinogen (tertiles) & $-.277 \pm .327$ & $.75(.39-1.43)$ & .391 \\
\hline
\end{tabular}




\section{Table 6}

Model Diagnostics: Cox Proportional Hazard model C-statistic for all cause midterm mortality, AIC, and Integrated Improvement Index.

\begin{tabular}{|l|l|l|}
\hline Risk markers & C-statistic & AIC \\
\hline Clinical model risk factors (rf) & .7072 & 485.01 \\
\hline Clinical model rf + eGFR & .7179 & 481.73 \\
\hline Clinical model rf + eGFR + albumin & .7986 & 352.35 \\
\hline Clinical model rf + eGFR + albumin + hsCRP > 5 mg/L & .8200 & 325.00 \\
\hline Clinical model rf + eGFR + albumin + hsCRP > 5 mg/L + sVCAM-1 & .8190 & 320.78 \\
\hline Integrated discrimination improvement $($ IDI) & Estimate & P-value \\
\hline IDI adding eGFR to clinical model rf & .03358 & .03284 \\
\hline IDI adding albumin to clinical model rf +eGFR & .06786 & .00048 \\
\hline IDI adding hsCRP to clinical model rf + eGFR + albumin & .08352 & .00005 \\
\hline IDI adding sVCAM to clinical model rf + eGFR + albumin + hsCRP > 5 mg/L & .01200 & .17352 \\
\hline
\end{tabular}

Clinical model risk factors (rf) include age, diabetes mellitus, coronary artery disease, critical limb ischemia. IDI P-value for the addition of sVCAM-1, sTNFR2, and fibrinogen $=$ NS.

AIC, Akaike Information Criterion 